\title{
Absolute-Scale Quantitative Energy Dispersive X-ray Analysis in Aberration- Corrected Scanning Transmission Electron Microscopy
}

\author{
Zhen Chen $^{1}$, Adrian J. D’Alfonso ${ }^{2}$, Matthew Weyland ${ }^{3,4}$, Daniel J. Taplin ${ }^{1}$, Scott D. Findlay ${ }^{1}$ and Leslie \\ J. Allen ${ }^{2}$ \\ 1. School of Physics and Astronomy, Monash University, Victoria 3800, Australia \\ 2. School of Physics, University of Melbourne, Victoria 3010, Australia \\ 3. Monash Centre for Electron Microscopy, Monash University, Victoria 3800, Australia \\ 4. Department of Materials Engineering, Monash University, Victoria 3800, Australia
}

Energy dispersive X-ray spectroscopy (EDX) has long been capable of determining elemental concentration ratios with a sensitivity of a few atomic percent at sub-micron resolution. This is achieved by comparison with reference specimens and taking signal ratios, thereby eliminating factors such as ionization cross section, fluorescence yield and detector geometry [1] that must otherwise be measured or calculated, and by avoiding low order zone axis orientations and the associated strong dynamical electron scattering ("channeling"). Atomic resolution EDX analysis in scanning transmission electron microscopy (STEM) has recently become possible [2-5] through improvements in aberration-corrected electron optics and X-ray detector design. However, channeling must be reckoned with in atomic resolution imaging since on-axis conditions are necessary for direct structure interpretation. Moreover, relative concentration may be less informative than the absolute number of atoms on this length scale.

In this talk we will demonstrate absolute-scale, quantitative agreement between the numbers of X-ray counts in experiment and first-principles simulations in STEM EDX using an atomically-fine electron probe [6]. $\mathrm{A} \mathrm{SrTiO}_{3}$ [001] test sample was used. The experiment was carried out using an aberrationcorrected FEI Titan ${ }^{3}$ electron microscope operating at $302 \mathrm{kV}$. A conventional Si(Li) detector was used, and obtaining good counting statistic necessitated averaging the EDX signal over several unit cells, forfeiting atomic-resolution information but gaining stability against noise and aberrations in the probeforming optics [7]. Simulations were carried out using the freely available $\mu$ STEM software package [8], incorporating calculation of the ionization cross-section and of channeling of the electron probe. For comparison with experiment it is further necessary to account for such factors as beam current, detector efficiency/geometry and fluorescence yields. Results are shown in figure 1 for a range of thicknesses and two different probe-forming aperture angles (15.2 $\mathrm{mrad}$ and $21.5 \mathrm{mrad})$.

An independent measurement of sample thickness was essential. Position-averaged convergent beam electron diffraction (PACBED) has been used for precision thickness determination [7]. Figure 2 shows a comparison between experimental and simulated PACBED patterns showing good visual agreement. To make this more objective, an $\mathrm{L}^{2}$-norm measure was used to compare simulated and experimental PACBED patterns. The central plot in figure 2 shows that such plots have a clear global minimum, which proved to be in good agreement with the visual approach. However, to obtain clear features in the PACBED pattern, we used a probe-forming aperture of $9.2 \mathrm{mrad}$, smaller than that used for the EDX experiments. An alternative approach is to quantify the intensity in the annular dark field (ADF) image [9] recorded simultaneously with the EDX image. The results of this approach are seen to be in excellent agreement with the PACBED method in the right hand graph in figure 2 [10]. 
References:

[1] JI Goldstein et al in "Principles of Analytical Electron Microscopy", ed. D.C. Joy et al, (Plenum Press, New York, 1986) p. 155.

[2] AJ D’Alfonso et al, Phys. Rev. B 81 (2010), 100101(R).

[3] M Chu et al, Phys. Rev. Lett. 104 (2010), 196101.

[4] PG Kotula et al, Microscopy and Microanalysis 18 (2012), p. 691.

[5] G Kothleitner et al, Phys. Rev. Lett. 112 (2014), 085501.

[6] Z Chen et al, Submitted for publication (2014).

[7] JM LeBeau et al, Ultramicroscopy 110 (2010), p. 118.

[8] LJ Allen et al, Ultramicroscopy in press (2015) doi:10.1016/j.ultramic.2014.10.011.

[9] JM LeBeau et al, Phys. Rev. Lett. 100 (2008), 206101.

[10] The authors thank Dr N.J. Zaluzec, Argonne National Laboratory, and A. Sandborg, EDAX Inc., for assistance with the geometry of our EDX detector. This research was supported by the Australian Research Council under the Discovery Projects funding scheme (Projects DP110102228 and 140102538), its DECRA funding scheme (Project DE130100739) and its LIEF funding scheme (Project LE0454166).
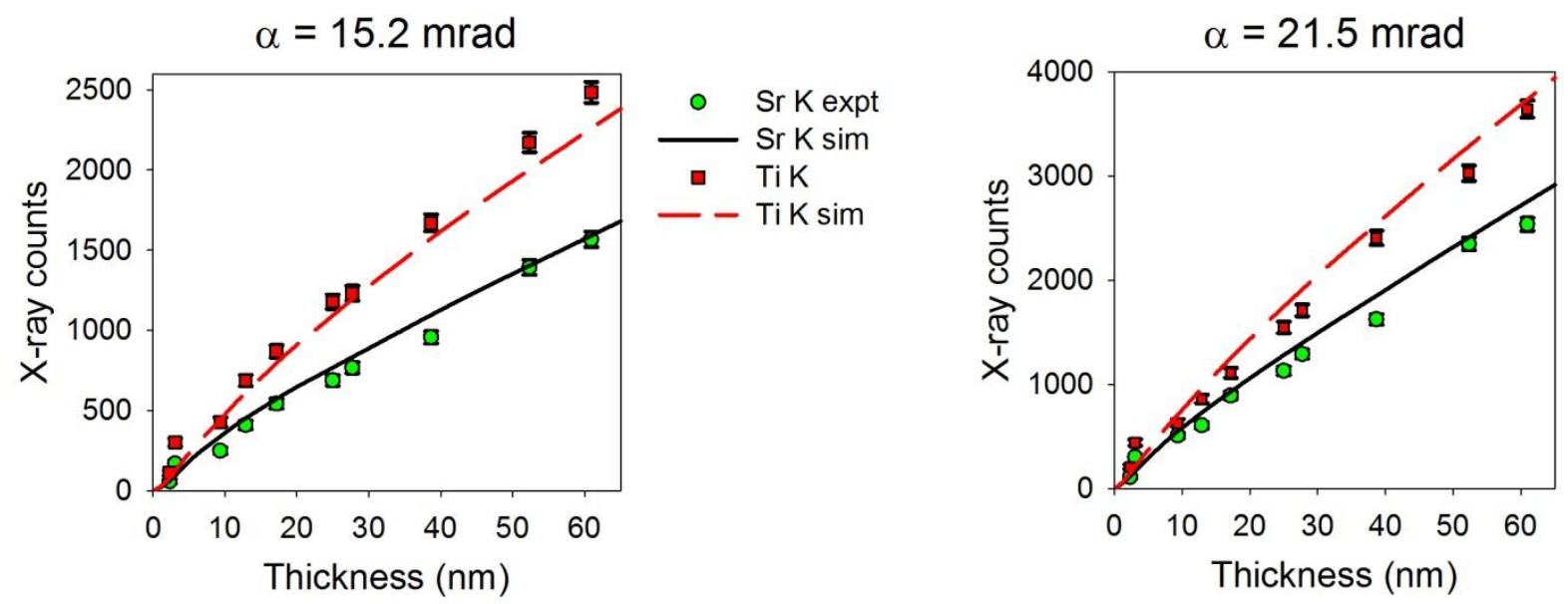

Figure 1. Absolute-scale comparison of X-ray counts between experiment and simulation for the $\mathrm{Sr} \mathrm{K}$ shell and Ti K-shell peaks for two different probe-forming aperture semiangles $\alpha$.
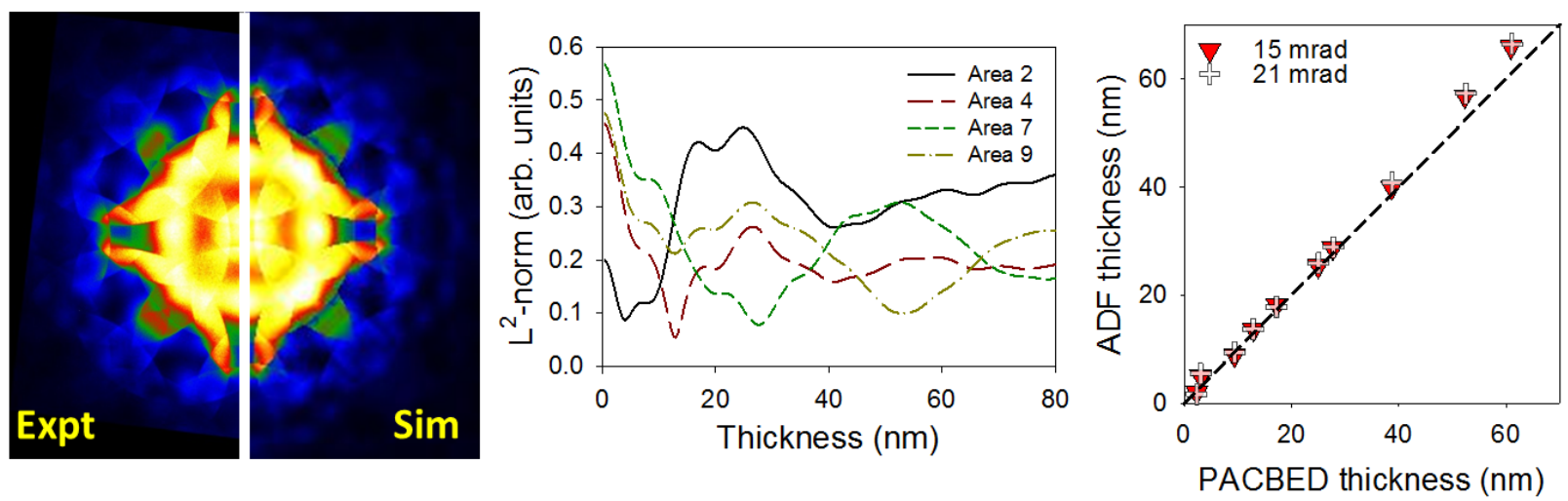

Figure 2. Left: Visual comparison of PACBED pattern between experiment and simulation $(\alpha=9.2$ mrad) (area 9). Centre: $\mathrm{L}^{2}$-norm analysis automating PACBED thickness determination. Right: Comparison of thicknesses determined by PACBED and by quantitative ADF STEM. 